\title{
Association Between Upstream Purine Complexes of Human Caveolin-1 Gene and Schizophrenia in Qazvin Province of Iran
}

\author{
Reza Najafipour ${ }^{1}$; Abolfazl Heidari ${ }^{1}$; Safar Ali Alizadeh ${ }^{1}$; Hannaneh Ghafelebashi ${ }^{2}$; Zahra \\ Rashvand ${ }^{1}$; Amir Javadi ${ }^{3}$; Mohammad Moradi ${ }^{4}$; Hosein Afshar ${ }^{4,}$ \\ ${ }^{1}$ Cellular and Molecular Research Center, Qazvin University of Medical Sciences, Qazvin, IR Iran \\ ${ }_{3}^{2}$ Tarbiat Modarres University, Tehran, IR Iran \\ ${ }^{3}$ School of Allied Medical Sciences, Tehran University of Medical Sciences, Tehran, IR Iran \\ ${ }^{4}$ Qazvin University of Medical Sciences, Qazvin, IR Iran \\ *Corresponding Author: Hosein Afshar, Qazvin University of Medical Sciences, Qazvin, IR Iran. Tel:+98-2833362959, +98-9121812000, Fax: +98-2833362960, E-mail: hoseinafshar1345@ \\ gmail.com
}

Received: June 25, 2014; Revised: September 27, 2014; Accepted: November 12, 2014

\begin{abstract}
Background: Caveolin is a multifunctional and scaffolding membrane protein, which involves cholesterol trafficking to plasma lipid microdomain. It organizes and targets synaptic parts of the neurotransmitter and neurotrophic receptor signaling pathways. Caveolins are encoded by CAV-1, 2 and 3 genes. Disruption of the CAV1 would likely ruin the neuronal signaling, which leads to symptoms of schizophrenia in predisposed individuals.

Objectives: The upper area of CAV-1 gene is highly conserved and can have a regulatory role in neurodegenerative diseases. This study was designed to find out the possible association of polymorphisms of this area and schizophrenia.

Patients and Methods: In a case-control study, 254 blood samples were obtained from 127 patients with schizophrenia and 127 well matched controls referred to 22 Bahman Hospital of Qazvin University of Medical Sciences (QUMS) in Qazvin province, Iran, using simple random sampling method. After extracting DNA, the upper region of the human CAV1-gene was amplified by PCR in all collected samples. The products were visualized by silver staining in $10 \%$ polyacrylamide gel and then sequenced.

Results: We detected nine homozygotes in patients and 15 in control subjects. Homozygosity was $7.08 \%$ and $11.8 \%$ in cases and control, respectively. Nine types homozygote haplotype were detected in upper region of the CAV1 gene in cases and controls. Three haplotypes were common in cases and controls; four haplotypes were seen in controls only and two in cases.

Conclusions: Our findings implied a significant correlation between some haplotypes of upper region of CAV1 gene and schizophrenia. Existence of some haplotypes and lack of another in CAV1 upstream can suggest a significant correlation between schizophrenia and some haplotypes.

Keywords: Caveolin-1; Schizophrenia; Iran
\end{abstract}

\section{Background}

Schizophrenia is a serious and neurodevelopmental disorder, which affects approximately $1 \%$ of the general population often-devastating effects such as psychological, social and financial skills. Disease symptoms include delusions, auditory hallucinations and thinking disorder. Schizophrenia can disturb memory, attention, thought and motivation of affected patients (1). Twin concordance rates, family and adoption studies, genetic linkage and allelic association analyses revealed a strong genetic element contributing to schizophrenia (2). The heritability of schizophrenia is estimated to be $80 \%$ (3). Therefore, due to the high percentage of heredity involvement, over 1000 genes have been tested to assess such an association. This makes schizophrenia one of the most studied disorders through a candidate gene approach $(4,5)$. Caveolins are encoded by CAV-1, 2 and 3 genes (6). Caveolins (encoded by CAV1 gene) are multifunctional scaffolding and cholesterol binding proteins organizing other lipids and proteins in surface domains; also they regulate various cellular functions such as lipid homeostasis, vesicular trafficking and signal transduction. In fact, Caveolin is extensively expressed in the nervous systems (7-11). In CAV1 knock-out mice, neurodegeneration and aging is more advanced than normal mice $(12,13)$. Current analyses of genomic structural variations in patients with schizophrenia showed that caveolin-1 gene (CAV1) is disrupted by an insertion mutation, therefore the CAV1 is identified as a rare structural variant correlated with schizophrenia (14). Given the importance of this region in neurodegenerative diseases, any changes in this area of genome may result in illnesses such as schizophrenia. CAV1 is also known as an interacting partner of G-protein and its loss can cause destruction of neuronal signaling leading to signs of schizophrenia in susceptible persons (9-11). 
NajafipourR et al.

\begin{tabular}{l}
\hline Table 1. The CAV1 Upstream Purine Complex in Different Species \\
\hline Gene Sequence \\
\hline Homo sapiens $\sim \mathbf{- 1 . 5} \mathbf{~ k b}$ \\
\hline GAAAGAAAAGAAAAAAGAAAAGGAAGGAAGGAAGGAAAGAAGGAAGGAAGGAAGGAAGGAAGGAAGGAAGGAAGGAAGGAAGGAAGGAAGGAAGGAAG \\
GAAGGAAAGAAAGAAGAAAGAAGAAAGAAAGAAAGAAAGAAAGAAAGAAAGAAAAGAAAGGAAGGAAGGAAGGAAGGGGAAGGAGGAAGA \\
Macaque primates -1.8 kb \\
GGAAAAAGAAAGAAAGAAAGAAAGAAAGAAAGAAAGAAAGAAAGAAAGAAAGAAAGAAAGAAAGAAAGAAAGAAAGAAAGAAAGAAAGAAAGAAAGAAAGA \\
AAGAAAGGAAGGAAGGAAGGAAGGATGTAAGGAAGGAAGGAAGGAAGGAAGAAAAAACAAAGAAAGAAAGAAAGAAAAAGAGAAA \\
Mouse -3.3 kb \\
GAGGAAGAAGAAGAAGAAGAAGAAGAAGAAGAAGAAGAAGAAGAAGAAGAAGAAGAAGAAGAAGAAGA \\
\hline
\end{tabular}

The purine rich complex at the $1.5 \mathrm{~kb}$ upper region of the human CAV1 gene is composed of three tandem STRs of GGAA, GAAA and GGAA motifs, which is conserved in different species as shown in Table $1(15,16)$. A skew in the homozygous haplotype of this region in patients with multiple sclerosis (MS) and late onset Alzheimer's disease (AD) has been reported versus controls. Twenty homozygous haplotypes have been identified in two neurodegenerative disorders increasing CAV1 gene expression (17).

\section{Objectives}

In the current study, test and trails were conducted and observed, to obtain a correlation between the genome area and schizophrenia.

\section{Patients and Methods}

In this case-control study, 127 unrelated patients with schizophrenia were included (diagnosed based on diagnostic and statistical manual of mental disorders, fourth edition, 1994, DSM-IV). Patients with IQ below 90, major depression, bipolar disorder and abuse of amphetamine and cannabis were excluded. Samples were selected from 22 Bahman Hospital, a psychology special hospital of Qazvin University of Medical Sciences (QUMS), Iran. The 22 Bahman hospitals is a referral psychology center, which has four sections with 100 beds. Patients were selected by a psychologist using consecutive sampling method from April 2012 to April 2013. Furthermore, 127 healthy subjects were selected as controls. The control subjects well matched with cases regarding gender, age and ethnicity. Cases and controls were 51\% males and 49\% females and their mean age was 41.5 years. A 5-milliliter blood sample was collected from individuals and stored at $-20^{\circ} \mathrm{C}$ until use. An informed consent was obtained from all participants and the study was approved in the ethics committee (Ethic no; 93.04.03-8890).The sample size was calculated according to this formula:

$\overline{\mathrm{F}=15 \%{ }_{\text {(loss to follow-up) }}, 1-\alpha=95 \%, 1-\beta=80 \%, \mathrm{P}_{0}=0.18, \mathrm{P}_{1}=0.35}$

\subsection{PCR and Sequencing}

All laboratory techniques were performed in molecular and cellular research center of QUMS. Five-milliliter blood samples were collected from patients and controls. DNA was extracted using DNA Extraction Kit (Roche; Germany). The PCR primers were designed by primer three tools for amplifying upstream region of the CAV1 gene. These primers were Forward; 5'-CAGGCTGCAGTGACCTATG-3' and Reverse; 5'-TCTGTACAACGAATCCCTGTG-3'. PCR reactions were performed in $25 \mu \mathrm{L}$ volume, including $100 \mathrm{ng}$ genomic DNA, 10 pmol of each primer,12.5 microliter Mastermix (Takara; Japan) and 10 microliters of distilled water. PCR temperature profiles were initially denaturized at $95^{\circ} \mathrm{C}$ for three minutes, 30 seconds at $95^{\circ} \mathrm{C}, 30$ seconds at $60^{\circ} \mathrm{C}, 30$ seconds at $72^{\circ} \mathrm{C}$, for 30 cycles, followed by five minutes final extension at $72^{\circ} \mathrm{C}$. These reactions were performed by a veriti $\mathrm{ABI}$ system. The produced fragments with 388 bp were detected on 10\% polyacrylamide gel by electrophoresis and silver staining. The homozygote amplicons on gel were selected for sequence analysis. The sequencing was performed by $3130 \mathrm{ABI}$ genetic analyzer. We looked for three tandem STRs of GGAA, GAAA, and GGAA motifs in sequenced fragments. All statistical analyzes were performed using SPSS software (version 16).

\section{Results}

After DNA extraction, upstream CAV1 gene was amplified by PCR with specific primers. All PCR products were sequenced to check this region. An example image of the sequences was shown in Figure 1. We detected nine homozygotes in patients with schizophrenia and 15 homozygotes in the control subjects. Homozygosity rates were $7.08 \%$ and $11.8 \%$ in cases and controls, respectively. Nine different types of homozygote haplotypes with different repeat numbers were detected in cases and controls. Some of homozygote haplotypes were detected in both patients and controls, but a number of haplotype lengths were found in cases and not observed in the controls and some of haplotype lengths were identified in controls and not observed in the cases. Details of results are shown in Table 2. These haplotypes contained 4-13-7 (110 bp), 5-10-8 (114 bp), 5-12-9 (118 bp) and 6-13-8 (110bp) (Table 3), while haplotypes 4-12-7 (106 bp), 7-11-9 (118 bp), 1112-6 (126 bp), 7-12-8 (124 bp) and 11,10,8 (130 bp) only existed in controls (haplotypes 6-9, Table 3). Only one haplotype was seen in cases only (haplotype n. 4, Table 3). One example of results is summarized in Tables 1 and 3. 
Najafipour Ret al.

Table 2. CAV1 Purine Complex Motif Lengths Observed in the Controls and Cases

\begin{tabular}{|c|c|c|c|c|}
\hline \multirow{2}{*}{$\begin{array}{l}\text { Variable Repeat (Repeat } \\
\text { Number) }\end{array}$} & \multicolumn{2}{|c|}{ Observed } & \multicolumn{2}{|c|}{ Frequency } \\
\hline & Case & Control & Case & Control \\
\hline \multicolumn{5}{|l|}{ GGAA } \\
\hline 4 & 1 & 5 & 0.11 & 0.33 \\
\hline 5 & 5 & 1 & 0.55 & 0.06 \\
\hline 6 & 3 & 1 & 0.33 & 0.06 \\
\hline 7 & 0 & 5 & 0 & 0.33 \\
\hline 11 & 0 & 3 & 0 & 0.2 \\
\hline \multicolumn{5}{|l|}{ GAAA } \\
\hline 10 & 2 & 2 & 0.22 & 0.13 \\
\hline 11 & 0 & 3 & 0 & 0.2 \\
\hline 12 & 3 & 7 & 0.33 & 0.46 \\
\hline 13 & 4 & 3 & 0.44 & 0.2 \\
\hline \multicolumn{5}{|l|}{ GGAA } \\
\hline 6 & 0 & 2 & 0 & 0.13 \\
\hline 7 & 1 & 5 & 0.11 & 0.33 \\
\hline 8 & 5 & 5 & 0.55 & 0.33 \\
\hline 9 & 3 & 3 & 0.33 & 0.2 \\
\hline
\end{tabular}

Table 3. CAV1 Purine Complex Haplotypes Observed in Controls and Cases

\begin{tabular}{|c|c|c|c|c|c|c|c|c|}
\hline \multirow[t]{2}{*}{ Haplotypes N. } & \multirow[t]{2}{*}{ GGAA (n1) } & \multirow[t]{2}{*}{ GAAA (n2) } & \multirow[t]{2}{*}{ GGAA(n3) } & \multirow[t]{2}{*}{ Length, bp } & \multicolumn{2}{|c|}{ Number of Homozygote } & \multicolumn{2}{|c|}{ Frequency } \\
\hline & & & & & Schizophrenia & Control & Schizophrenia & Control \\
\hline 1 & 4 & 13 & 7 & 110 & 1 & 2 & 0.11 & 0.13 \\
\hline 2 & 4 & 12 & 7 & 106 & 0 & 3 & 0 & 0.2 \\
\hline 3 & 5 & 10 & 8 & 114 & 2 & 1 & 0.22 & 0.06 \\
\hline 4 & 5 & 12 & 9 & 118 & 3 & 0 & 0.33 & 0 \\
\hline 5 & 6 & 13 & 8 & 110 & 3 & 1 & 0.33 & 0.06 \\
\hline 6 & 7 & 11 & 9 & 118 & 0 & 3 & 0 & 0.2 \\
\hline 7 & 11 & 12 & 6 & 126 & 0 & 2 & 0 & 0.13 \\
\hline 8 & 7 & 12 & 8 & 124 & 0 & 2 & 0 & 0.13 \\
\hline 9 & 11 & 10 & 8 & 130 & 0 & 1 & 0 & 0.06 \\
\hline Total & - & - & - & - & 9 & 15 & - & - \\
\hline
\end{tabular}

Figure 1. Homozygote Haplotype in a Patient With Schizophrenia

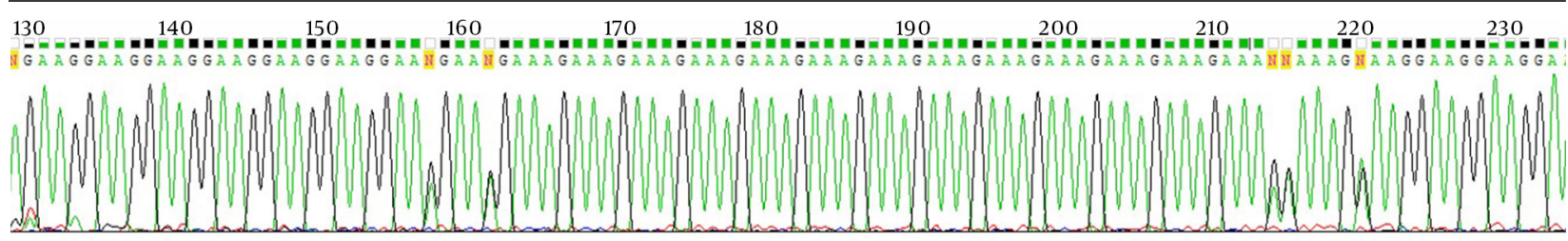

This figure shows tandem STRs of GGAA, GAAA, GGAA motifs and haplotype length [e.g. 126-bp (11-12-6 haplotype)].

\section{Discussion}

There are a number of reports about implicating purine complex of upstream CAV1 human gene involvement in pathophysiology of neurodegenerative diseases such as multiple sclerosis (MS) and Alzheimer's disease (AD) $(8,18)$. According to existing information, this region is conserved across several species, and consists of 
GGAA, GAAA and GGAA motifs, transcription consensus sites for the Ets and IRF (interferon regulatory factor) family members. The interaction between Ets and IRF family members has been shown in different studies (7, $8)$. Furthermore, inflammation plays a role in neurodegenerative diseases and several inflammatory transcription factors like STAT4 and interferon regulatory factor (IRF). In which, these factors bind to the CAV1 upstream purine complex and regulate target genes $(12,19,20)$. Some literatures categorized Schizophrenia as a neurodegenerative disease $(1,14)$. To examine the hypothesis that purine complex of upstream CAV1 may be related to pathogenesis of schizophrenia, we investigated this region in a group of patients with schizophrenia and compared the results with well-matched controls. In this research, homozygote haplotypes of GGAA, GAAA and GGAA motifs were compared between cases and controls. Some haplotypes were found in patients only. Chi square test showed that these haplotypes significantly related to object (e.g. haplotype 4 , Table $3, \mathrm{P}=0.016, \mathrm{P}<$ 0.05). Nevertheless, some haplotypes were found in controls only. Statistical analysis showed no significant association between these haplotypes and schizophrenia (for example; haplotype 6, Table 3, $\mathrm{P}=0.15,7$ and $8 \mathrm{P}=$ $0.25, \mathrm{P}>0.05)$. These findings implied a significant correlation between schizophrenia and some haplotypes of this region of CAV1 gene. Existence of some haplotypes and lack of another in CAV1 upstream can suggest a significant correlation between schizophrenia and some haplotypes. To the best of our knowledge, this was the first study to assess the association between purine complex upstream region of human CAV1 and schizophrenia disease. These results could be useful for further researches in this field.

\section{Acknowledgements}

The authors would like to kindly thank Taghi Naserpour, the chief of Cellular and Molecular Research Center of Qazvin University of Medical Sciences, for his help and technical support. Besides, we want to thank the research deputy of Qazvin University of Medical Sciences for financial support and reference laboratory of Qazvin University of Medical Sciences for technical support.

\section{Authors' Contributions}

All authors cooperated in design, conduction, data gathering, data analysis and writing the manuscript. All authors read and approved the manuscript.

\section{Funding/Support}

This manuscript was supported by the research deputy of Qazvin University of Medical Sciences (QUMS), grant no 92-11-07-561.

\section{References}

1. Karoutzou G, Emrich HM, Dietrich DE. The myelin-pathogenesis puzzle in schizophrenia: a literature review. Mol Psychiatry. 2008;13(3):245-60.

2. Kirov G, O'Donovan MC, Owen MJ. Finding schizophrenia genes. J Clin Invest. 2005;115(6):1440-8.

3. Tsuang MT, Faraone SV. Genes, environment and schizophrenia. BrJ Psychiatry. 2001;178(40):s18-24.

4. Owen MJ, Gottesman II, McGuffin P. Psychiatric Genetics and Genomics.Oxford: Oxford University Press; 2002.

5. National Institute of Mental Health.. SZ Gene (SchizophreniaGene) Field Synopsis of Genetic Association Studies in SZ. 2011. Available from: http://www.szgene.org.

6. Shin T, Kim H, Jin JK, Moon C, Ahn M, Tanuma N, et al. Expression of caveolin-1, -2 , and -3 in the spinal cords of Lewis rats with experimental autoimmune encephalomyelitis. J Neuroimmunol. 2005;165(1-2):11-20.

7. Kanno Y, Levi BZ, Tamura T, Ozato K. Immune cell-specific amplification of interferon signaling by the IRF-4/8-PU.1 complex. $J$ Interferon Cytokine Res. 2005;25(12):770-9.

8. Heidari A, Behmanesh M, Sahraian MA, Meshkani R, Darvish H, Najmabadi $\mathrm{H}$, et al. The human caveolin 1 gene upstream purine complex and neurodegeneration--a common signature. J Neuroimmunol. 2011;236(1-2):106-10.

9. Allen JA, Halverson-Tamboli RA, Rasenick MM. Lipid raft microdomains and neurotransmitter signalling. Nat Rev Neurosci. 2007;8(2):128-40.

10. Francesconi A, Kumari R, Zukin RS. Regulation of group metabotropic glutamate receptor trafficking and signaling by the caveolar/lipid raft pathway. J Neurosci. 2009;29(11):3590-602.

11. Boulware MI, Kordasiewicz H, Mermelstein PG. Caveolin proteins are essential for distinct effects of membrane estrogen receptors in neurons. J Neurosci. 2007;27(37):9941-50.

12. Musikacharoen T, Oguma A, Yoshikai Y, Chiba N, Masuda A Matsuguchi T. Interleukin-15 induces IL-12 receptor beta1 gene expression through PU.1 and IRF 3 by targeting chromatin remodeling. Blood. 2005;105(2):711-20.

13. Head BP, Peart JN, Panneerselvam M, Yokoyama T, Pearn ML, Niesman IR, et al. Loss of caveolin-1 accelerates neurodegeneration and aging. PLoS One. 2010;5(12).

14. Walsh T, McClellan JM, McCarthy SE, Addington AM, Pierce SB Cooper GM, et al. Rare structural variants disrupt multiple genes in neurodevelopmental pathways in schizophrenia. Science. 2008;320(5875):539-43.

15. Bhatnagar A, Sheffler DJ, Kroeze WK, Compton-Toth B, Roth BL Caveolin-1 interacts with 5-HT2A serotonin receptors and profoundly modulates the signaling of selected Galphaq-coupled protein receptors. J Biol Chem. 2004;279(33):34614-23.

16. Heshmati Y, Mirabzadeh A, Feizzade G, Gilanipour M, Etminan MR, Khoram Khorshid HR, et al. A novel polymorphic purine complex at the $1.5 \mathrm{~kb}$ upstream region of the human caveolingene and risk of Alzheimer's disease; extra-short alleles and accumulated allele homozygosity. Am J Med Genet B Neuropsychiatr Genet. 2009;150B(2):248-53.

17. Zarif Yeganeh M, Ghaffarpour M, Farhud DD, Karimlou M, Ghabaee M, Haghighi Nazari A, et al. Skew in the human caveolin 1 gene upstream purine complex homozygote haplotype compartment in multiple sclerosis. J Neuroimmunol. 2009;216(12):103-7.

18. Cohen AW, Hnasko R, Schubert W, Lisanti MP. Role of caveolae and caveolins in health and disease. Physiol Rev. 2004;84(4):1341-79.

19. Chiarini A, Whitfield J, Bonafini C, Chakravarthy B, Armato U, Dal Pra I. Amyloid-beta(25-35), an amyloid-beta(1-42) surrogate, and proinflammatory cytokines stimulate VEGF-A secretion by cultured, early passage, normoxic adult human cerebral astrocytes. J Alzheimers Dis. 2010;21(3):915-26.

20. Vandenbroeck K, Urcelay E, Comabella M. IFN-beta pharmacogenomics in multiple sclerosis. Pharmacogenomics. 2010;11(8):1137-48. 